\title{
Las necesidades sociales como eje de la formación médica
}

Jaime Arturo Gómez Correa(a)

Liliana Villa Vélez ${ }^{(b)}$

Miglena Kambourova ${ }^{(c)}$

\section{Introducción}

\section{Antecedentes curriculares}

El currículo tradicional de la Facultad de Medicina de la Universidad de Antioquia se reformó en 2000 considerando los cambios para la época en la pedagogía y la educación médica a nivel nacional e internacional. Son sus principios pedagógicos: la formación integral y la investigación formativa; los curriculares: la flexibilidad, la interdisciplinariedad y la pertinencia; los didácticos: la solución de problemas, el paradigma cognitivo y las didácticas activas ${ }^{1}$. Para valorar la puesta en práctica del currículo se realizó una investigación entre 2009 y 2012; los resultados demostraron que sí bien se ha logrado avanzar en la materialización de los principios fundamentales aún hay muchos aspectos por mejorar, algunos de los cuales se describen a continuación.

Persiste la separación entre básicas y clínicas, situación que se expresa igualmente en la división entre teoría y práctica; no se avanzó en la creación de nuevos entornos de aprendizaje como los espacios en donde la gente desarrolla su vida (familia, barrios) lo que afecta la pertinencia, y por el contrario se profundizó la enseñanza en hospitales de alta complejidad, siguiendo la inercia del modelo de salud prevalente en el país.

Aunque se avanzó en procesos de aprendizajes activos, se acepta que falta por desarrollar "la autonomía a partir de espacios educativos democráticos para adquirir, cuestionar y aplicar el conocimiento como una respuesta a las necesidades académicas y sociales"2 (p. 215), afirmación que también permite inferir deficiencias en el área de la investigación formativa.

La interdisciplinariedad como principio no ha logrado pasar del papel a la realidad, y lo único que se ha logrado es solo un abordaje multidisciplinario de los principales problemas de salud, en gran parte, porque muchos profesores están aferrados a una medicina disciplinar desde su formación como especialistas o sub especialistas.

Todo lo anterior motiva que se desarrollen propuestas que permitan superar las dificultades del modelo de formación actual.

(a,b) Departamento de Medicina Preventiva, Facultad de Medicina, Universidad de Antioquia.

Medellín, Antioquia,

Colombia. Carrera 51

D N $62-29,21960$

41. jaime.gomez@ udea.edu.co;

liliana.villa@udea.edu.co

(c) Departamento de

Educación Médica,

Facultad de Medicina, Universidad de

Antioquia. Medellín,

Antioquia, Colombia miglena.kambourova@ udea.edu.co 


\section{La experiencia}

Desde el año 2004, por invitación de líderes del asentamiento Altos de Oriente (sector perteneciente a la vereda Granizal), se inició una relación entre el Departamento de Medicina Preventiva de la Facultad de Medicina y los pobladores del asentamiento, que se ha ido fortaleciendo a través del tiempo y se ha ampliado a los otros sectores de la vereda.

La vereda Granizal del municipio de Bello, con cerca de 20.000 personas es considerada como el segundo mayor poblado receptor de población desplazada en el país y el primero en el Área Metropolitana del Valle de Aburrá3; está constituida por seis asentamientos: Manantiales, El Pinar, Oasis de Paz, El regalo de Dios, El siete, Altos de Oriente 1 y Altos de Oriente 2. En una caracterización sociodemográfica de los asentamientos "Altos de Oriente 1 y 2 " se encuentra que en el $80 \%$ de casos, la familia o alguno de los integrantes había sido desplazado por la violencia, lo cual indica que se trata de un asentamiento constituido en su mayoría por víctimas de la violencia y que el $42 \%$ de la familias tenía una Necesidad Básica Insatisfecha (NBI) y un $45 \%$ tenía dos o más NBI ${ }^{4}$. Ello coincide con la declaración de la corte constitucional de que las víctimas de la violencia se encuentran en un "estado de cosas inconstitucional..." efecto de la desatención y el abandono por parte de las instituciones gubernamentales 5 .

En los últimos cinco años aquella relación se ha formalizado e institucionalizado, mediante la formulación y ejecución de proyectos de extensión financiados en su mayoría por el Banco Universitario de Programas y Proyectos (BUPPE) de la vicerrectoría de extensión de la Universidad de Antioquia. Los diversos proyectos se han orientado a responder a problemas y necesidades identificados por los mismos pobladores, como parte de la función universitaria de extensión solidaria. Ellos son:

- "Caracterización demográfica y social del asentamiento Altos de Oriente" ${ }^{4}$ que ha servido de línea de base para distintos proyectos en la vereda; realizado en 2010.

- "Evaluación integral de niños de hogares comunitarios del Instituto Colombiano de Bienestar Familiar (ICBF)" que además del servicio que se presta a la comunidad se ha convertido en un curso formal del currículo y se desarrolla desde 2012.

- "Proceso de formación y acompañamiento psicosocial para el fortalecimiento de líderes y lideresas de la vereda Granizal, municipio de Bello" que ha tenido impactos importantes en la organización y la participación social en la vereda y se ejecutó con recursos de donantes privados en 2012.

. "Atención Primaria en Salud" con cobertura sobre 100 familias iniciado desde finales del año 2013.

. "Escuela de formación de gestores comunitarios para la acción con énfasis en desarrollo local" la cual fue diseñada por iniciativa y con participación de los mismos líderes, con financiación y participación del Programa de Naciones Unidas para el Desarrollo (PNUD) y las Facultades de derecho y medicina de la Universidad de Antioquia, ejecutado en 2014.

En perspectiva de lograr la articulación de las diferentes funciones misionales (docencia, investigación y extensión) los proyectos de extensión mencionados han concebido desde su diseño la inclusión de actividades de docencia e investigación.

La articulación con la docencia se ha logrado mediante la participación de los estudiantes en actividades de los proyectos, las cuales hacen parte del proceso de formación como profesionales de la salud. Así se estructuran cursos a partir de la realidad comunitaria, y desde ésta se promueve la reflexión teórica; los estudiantes participan en la realización de actividades, tales como, diseño y diligenciamiento de instrumentos de recolección de información, evaluación médica de beneficiarios de los proyectos, desarrollo de actividades de capacitación dirigidas a los grupos poblacionales beneficiarios, acompañamiento y asesoría a las familias en la solución de sus problemas de salud y afiliación al Sistema General de Seguridad Social en Salud.

El proyecto "Evaluación integral de niños de hogares comunitarios del ICBF" es clave en esta experiencia porque ha permitido integrar tres cursos formales del sexto semestre (Niñez I, Salud y Sociedad III y Epidemiología Clínica I) con el trabajo comunitario, como respuesta a una necesidad de las madres usuarias de los hogares comunitarios del ICBF, quienes debían presentar los certificados de 
salud de sus hijos como requisito para su matrícula, pero que les quedaba difícil conseguirlos por las barreras de acceso geográfico, administrativas y económicas.

A lo largo del tiempo se ha logrado consolidar el grupo de docentes participantes, mejorando la integración de contenidos y didácticas de los cursos; también la comunidad reconoce y demanda la presencia de la Universidad en sus espacios; además, los estudiantes matriculados en los cursos mencionados y quienes han participado en la experiencia, por medio de las evaluaciones afirman que la visita del lugar les permite tratar los verdaderos problemas de salud, conectar la teoría con la práctica, servir a la comunidad, crecer en valores y humanismo y aprender para la vida. El Cuadro 1 describe el número de actores involucrados.

Cuadro 1. Actores involucrados en la experiencia

\begin{tabular}{|l|c|c|c|}
\hline Semestre & Número de estudiantes de medicina* $^{*}$ & Niños evaluados en la comunidad** $^{*}$ & Docentes participantes $^{* * *}$ \\
\hline $2012-1$ & 20 & $241^{\#}$ & 4 \\
\hline $2012-2$ & 17 & $201^{\#}$ & 4 \\
\hline $2013-1$ & 16 & 119 & 5 \\
\hline $2013-2$ & 18 & 121 & 5 \\
\hline $2014-1$ & 18 & 120 & 5 \\
\hline $2014-2$ & 20 & 114 & 5 \\
\hline $2015-1$ & 23 & 110 & 5 \\
\hline
\end{tabular}

"Eventualmente han asistido estudiantes de último año de formación de pregrado en medicina y residentes de pediatría; "El mismo niño puede ser evaluado en varios semestres. Cada niño asiste con un adulto cuidador a las actividades; '" Los docentes son: pediatra, psicólogo, comunicadora, médico salubrista y médico epidemiólogo; ${ }^{\#}$ Número aproximado debido a que no se implementó un registro preciso

La experiencia comprende además un componente de construcción de conocimiento de la realidad de una comunidad particular, dando lugar a procesos de investigación con pertinencia. Los diferentes proyectos desde su diseño han estructurado un sistema de captura, procesamiento y análisis de información socio-económica y de carácter familiar e individual como: NBI, estructura familiar, nivel educativo de los integrantes, antecedentes prenatales, perinatales, crecimiento y desarrollo de los niños, estado de salud, entre otros. Este tipo de investigación aporta tanto a la construcción de equidad, porque permite visualizar realidades que generalmente permanecen ocultas, como al empoderamiento de las comunidades en la medida en que pueden utilizar la información para conocer su realidad y demandar solución de sus problemas.

\section{Reflexión pedagógica sobre la experiencia}

La experiencia descrita permite avanzar en el diseño y puesta en práctica de un nuevo currículo para la formación de los profesionales de medicina en los siguientes aspectos: nuevos entornos de aprendizaje e integración de las áreas básicas y las clínicas; el estudiante como protagonista de su aprendizaje reforzando el desarrollo de la competencia "aprender a aprender"; la experiencia como parte central del proceso de un aprendizaje significativo; la formación de una actitud investigativa y problematizadora mediante un pensamiento crítico que genera autonomía y creatividad; los problemas y las necesidades de la comunidad como base para estructurar el currículo; la interdisciplinariedad que favorece la integración de diferentes áreas del conocimiento para resolver las necesidades sociales; el desarrollo de una conciencia ético-política y valores de los sujetos en formación. 
El currículo de la Facultad de Medicina de la Universidad de Antioquia propone al estudiante como agente activo de su propio aprendizaje, lo cual se logra por medio de la aplicación de estrategias didácticas activas como la solución de problemas simulados o reales, el estudio de casos, el aprendizaje basado en problemas (ABP), los proyectos de aula, las visitas domiciliarias familiares ${ }^{6}$ y el trabajo en las comunidades, entre otras. Sin embargo, aún se imparte la enseñanza desde la pedagogía tradicional donde se exigen aprendizajes memorísticos y la pasividad estudiantil.

Lo vivido por los estudiantes en la vereda Granizal contrasta estas falencias y es un aporte más a las estrategias activas. Los contenidos teóricos y la exposición del profesor no son la prioridad porque el estudiante fuera del salón de clase se relaciona directamente con los problemas de las comunidades y los servicios de salud, buscando soluciones, articulando la práctica y la teoría, lo cual favorece la transferencia de los aprendizajes. La información que el estudiante encuentra es puesta a prueba en la práctica generando una transformación de la realidad con la que inicialmente se había encontrado ${ }^{7}$. La integración entre práctica y teoría permite superar la clásica división entre el ciclo básico (teoría) y el clínico (práctica), favoreciendo su integración en un currículo conformado por módulos de problemas que se abordan en la realidad de la vida de las comunidades o de los servicios de $\operatorname{salud}^{8} \mathrm{y}$ en la cual los conocimientos "básicos" sirven para interpretar y resolver los problemas de la "clínica"7.

Desde esta perspectiva emerge la necesidad de "aprender a aprender", que implica la capacidad de reflexionar en la forma en que se aprende y actuar en consecuencia, autorregulando el propio proceso de aprendizaje mediante el uso de estrategias flexibles y apropiadas que se transfieren y adaptan a nuevas situaciones ${ }^{9}$. Ante los problemas que visualiza, el estudiante desarrolla destrezas para buscar y seleccionar de manera crítica datos e información que sean relevantes para la interpretación de la realidad con la que entra en contacto. En esta época en que abunda la información es necesario seleccionarla y actualizarla con criterio; por ello es relevante asegurar el "aprender a aprender", más que garantizar el aprendizaje de unos conocimientos, generalmente transmitidos verticalmente por un docente o aprendidos de memoria de un texto y que en corto tiempo podrán ser obsoletos ${ }^{10}$.

El profesor universitario hoy en día no ocupa la centralidad en el proceso pedagógico; sin embargo, su labor continúa siendo esencial en la formación. Éste tiene un rol de acompañamiento a los estudiantes, con el fin de ofrecerles estrategias que faciliten su aprendizaje y los preparen para desenvolverse como profesionales en el mundo de la vida cotidiana. El profesor parte de su propia experiencia para llevar al estudiante a escenarios adecuados de aprendizaje como los espacios comunitarios. Como bien lo afirman Marín y Tamayo:

... es necesario comprender cómo el educador y el educando son sujetos activos, comprometidos con el acto educativo; no puede concebirse un educando inactivo, simple receptor de contenidos y mucho menos como sujeto inoperante; la concepción con la que se cuenta versa sobre un educando activo, comprometido, con un potencial cognitivo que le provee habilidades analíticas, criticas, sintéticas y con un potencial creador que le permite variar, transformar, co-producir y activar el mundo de la vida. ${ }^{11}$ (p. 16)

Esta experiencia propone una forma de vincular la escuela y la vida a partir de la intervención de problemas y necesidades sociales, que materializa la consigna "aprender haciendo", lo que implica la inversión del orden tradicional del aprendizaje que se basa en impartir primero la teoría, para su verificación posterior en la práctica. Los modelos pedagógicos de la escuela nueva ${ }^{12}$, asumen que la resolución de problemas prácticos por los propios medios de los estudiantes, desarrollan competencias que les serán útiles para su desempeño en la vida cotidiana, para lo cual habría que transformar el aula de clase en un espacio que guarde continuidad con el mundo exterior, lo cual hace a los estudiantes más activos y además fortalece la autonomía y el trabajo en equipo ${ }^{13}$.

La práctica propone entornos de aprendizaje diferentes a los tradicionales tales como los hogares, las familias y la comunidad; demanda una articulación con las redes de servicios de salud, empezando por los primeros niveles de atención del área de influencia en la comunidad; favorece una inserción temprana del estudiante en la resolución de los problemas de la comunidad, que se convierten en el eje y motor del proceso de aprendizaje. 
Al acercarse a la comunidad el estudiante llega con algunos conocimientos los cuales se ponen a prueba al enfrentar la realidad; cada uno de ellos valora su saber previo y de acuerdo con sus necesidades e intereses generados por la situación particular de las personas, conecta la nueva información con sus pre saberes con lo que se activan cambios en su estructura cognitiva, logrando el aprendizaje significativo; a éste se refiere Ausubel como aquel en que la nueva información adquiere significados para el aprendiz, por interacción con alguna información relevante ya existente en su estructura cognoscitiva, con un cierto grado de estabilidad, claridad, organización y diferenciación ${ }^{14}$.

A medida que el estudiante logra transferir los conocimientos a situaciones problemáticas, como son las que se presentan en la interacción con las comunidades,

se eleva su capacidad de aprender a aprender, se despierta el espíritu investigativo y crítico al comparar teoría con realidad, se desarrollan sus capacidades comunicativas, se sensibiliza con su compromiso social, se crean condiciones para aportar desde la subjetividad a las múltiples miradas de una misma realidad, lo cual favorece la intersubjetividad. ${ }^{6}$ (p. 650)

En la relación con la comunidad, se favorece el desarrollo de competencias comunicativas con pacientes, docentes, comunidad y entre los mismos estudiantes; la interacción se da de persona a persona en la triada estudiante - docente - comunidad; se genera un diálogo de saberes para la comprensión del contexto y se motivan los procesos de reflexión sobre la experiencia de aprendizaje.

El aprendizaje activo es determinante para desarrollar competencias investigativas, las cuales se pueden lograr desde los procesos iniciales de formación, pues al estudiante se le promueve la formulación de preguntas y la búsqueda activa de las respuestas. Esto permite la construcción del conocimiento sobre la realidad misma de las personas y comunidades, a partir de descripciones y sistematizaciones de dicha realidad, proponiendo una perspectiva diferente a la tradicional impuesta por la ciencia positivista hegemónica. En este sentido podríamos hablar de que se tienen en cuenta las necesidades locales, regionales y nacionales para dar respuesta a las problemáticas de cada contexto ${ }^{15,16}$.

Es así como la investigación formativa, uno de los principios pedagógicos del currículo de la Facultad, toma importancia en el proceso de formación, a través del aprendizaje por descubrimiento y construcción ${ }^{17}$. El estudiante se convierte en investigador desde una dinámica inductiva, que partiendo de las especificidades sociales, construye un conocimiento local y permite que los actores involucrados investiguen, aprendan y se transformen en dicha relación.

Un currículo se organiza y desarrolla desde las necesidades sociales. Según Marín y Tamayo ${ }^{11}$ citando a Torres:

Es necesario que éste sirva para atender a las necesidades que los alumnos y alumnas tienen de comprender la sociedad en la que les ésta tocando vivir y, por consiguiente, que favorezca el desarrollo de destrezas diversas, tanto técnicas como sociales, que les ayuden en su localización dentro de la comunidad como personas autónomas, críticas, democráticas y solidarias. (p. 63)

Un currículo cuyos contenidos se organizan por problemas de la vida real, permite trascender los currículos que se estructuran sobre asignaturas, materias o disciplinas, de forma lineal, con bajo nivel de integración, donde hay una fragmentación excesiva del conocimiento sin relación entre las distintas disciplinas. Algunos más evolucionados se estructuran sobre problemas de conocimiento o proyectos $^{18}$, procurando una mayor pertinencia de los objetos de aprendizaje y más protagonismo de los estudiantes para la construcción de aprendizajes significativos. Infortunadamente con frecuencia estos problemas o proyectos se abstraen del contexto para ser utilizados en el aula bajo "ambientes controlados", convirtiéndolos en simulaciones que pierden la esencia interactiva con la realidad, reduciendo las potencialidades creativas y dinamizadoras que ofrecen aquellos problemas propios de la vida cotidiana; en ese sentido se debe "orientar la metodología de la enseñanza hacia la solución de problemas concretos del mundo real, en vez de abrumar a los estudiantes con un curso detrás de otro, poco o nada vinculado con la realidad"19 (p. 19). 
La formación en escenarios reales favorece la interdisciplinariedad y sobre todo la transdisciplinariedad, sin que ello implique anular la disciplinariedad o la especificidad, como plantea Vaideanu:

... por el contrario, derriba las barreras entre disciplinas y pone de relieve la complejidad, la globalidad y el carácter sumamente imbricado de la mayoría de los problemas concretos a resolver. Ofrece así una visión más clara de la unidad del mundo, de la vida y de las ciencias. La interdisciplinariedad supone naturalmente la existencia de las disciplinas y reconoce que el enfoque disciplinario es muchas veces insustituible pero que al mismo tiempo dicha metodología suele resultar ya insuficiente en gran número de situaciones, y por ello, pone en cuestión los "contenidos parcelarios" y la existencia de barreras demasiado rígidas entre disciplinas. ${ }^{20}$ ( $\mathrm{p}$. 537-8)

Esta propuesta aporta a la formación con pertinencia social pues el estudiante se familiariza con los problemas de salud cotidianos de las personas, que representan un gran porcentaje de las dificultades de salud de la población en los lugares donde transcurre su vida. Adicionalmente, permite avizorar un reordenamiento de las funciones misionales de la universidad, puesto que la dinámica universitaria parte en este modelo de la acción transformadora sobre la realidad social de una comunidad (extensión), que permite articular y estructurar proyectos de aula en que los docentes y estudiantes enseñan y aprenden (docencia) y a partir de allí se genera conocimiento (investigación); esta forma de relacionamiento potencialmente reformula la actual consigna universitaria "La investigación y la docencia constituyen los ejes de la vida académica de la Universidad y ambas se articulan con la extensión para lograr objetivos institucionales de carácter académico o social"21 (p. 4), pues pone el énfasis del quehacer universitario en la transformación de la realidad social (extensión) y a partir de allí se dinamizan las otras funciones misionales.

La interacción del estudiante con el contexto, la comunidad y sus compañeros le permite desarrollar diferentes habilidades fundamentales para la vida y para la profesión, tales como, la auto-reflexión, pues pone al sujeto en relación con otro y con un contexto, lo cual le problematiza y le exige pensarse a él y al otro como seres distintos merecedores de mutuo respeto; en consecuencia se obliga a ponerse en el lugar de otro, pues reconoce que hay personas que viven en distintas condiciones a las propias, favoreciendo un sentido de compasión por las necesidades del prójimo ${ }^{13}$, evitando su instrumentalización, todo lo cual redunda en el otorgamiento de la dignidad a la vida propia y a la ajena.

A partir de las experiencias comunitarias se "humanizan" los estudiantes, en la medida en que se forman y transforman; se entiende esta humanización como sensibilización sobre las problemáticas sociales. Se logran visualizar otras realidades sabidas pero no conocidas, que comprenden los procesos de salud en relación estrecha con las problemáticas sociales; en este sentido se genera un impacto en la forma como los estudiantes se relacionan consigo mismos, con el otro y con el mundo.

La experiencia privilegia el desarrollo de valores y actitudes como la responsabilidad, el compromiso social, la ética, el trabajo en equipo, el aprender a aprender y la comunicación. En los estudiantes se despiertan sentimientos de empatía hacia las familias y los niños, se mejora la relación médico paciente y se da un nuevo significado a su quehacer como ser humano y como médico integral.

\section{Conclusiones}

Es necesario avanzar en la estructuración de un currículo integrado que fundamente los procesos de aprendizaje sobre los problemas más frecuentes que la sociedad presenta en una realidad concreta. Este fundamento exige el desarrollo de los aprendizajes en los lugares donde se presentan los problemas sociales (el barrio, la calle, la familia, el trabajo); ello hace que el aprendizaje sea pertinente y lo hace significativo; difumina las divisiones entre teoría y práctica y entre básicas y clínicas, pues son las problemáticas que aborda la universidad y el estudiante desde los primeros semestres, las que 
demandan la pesquisa y la investigación para su solución, acudiendo al conocimiento de una forma compleja e interdisciplinaria como son los problemas de la vida real.

En este orden de ideas, la experiencia presentada aporta a la construcción de un currículo integrado en el programa de medicina, ya que desarrolla un enfoque de los procesos enseñanza y aprendizaje que permite un impacto en distintas esferas de la vida universitaria y social. En lo universitario exige interdisciplinariedad del conocimiento; posibilita construir conocimientos locales y por ende desarrollar una investigación que se aplica a los problemas de la realidad local; la formación del estudiante se torna más pertinente y contextualizada; promueve flexibilidad tanto en los programas como en los mismos estudiantes y docentes, dado que los contenidos se enriquecen con los problemas mismos de la sociedad; en lo social la universidad aporta todo su conocimiento a la transformación para mejorar las condiciones de vida, revalorando la interacción universidad-sociedad.

Vale la pena mencionar que este trabajo parte de la perspectiva de algunos de los docentes, en la medida en que reflexionan sobre la importancia del trabajo en la comunidad y su impacto en la formación de los estudiantes de medicina. Es necesario que a futuro se desarrollen otras reflexiones e investigaciones desde el punto de vista de los demás actores implicados como los estudiantes y la comunidad.

\section{Colaboradores}

Los autores participaron igualmente en todas las etapas de elaboración del artículo.

\section{Agradecimientos}

A todos los actores que han posibilitado el desarrollo de esta experiencia: habitantes de la vereda Granizal, vicerrectoría de extensión, Facultad de Medicina, y de manera especial a los docentes Miriam Bastidas, Margarita Gómez y Federico Ordoñez que han participado en el acompañamiento al proceso de formación de profesionales en la comunidad.

\section{Referencias}

1. Comité de Currículo. La visión curricular, los propósitos de formación, la propuesta pedagógica: para la renovación curricular aspectos conceptuales. Medellín: Universidad de Antioquia, Facultad de Medicina; 1999.

2. Galindo LA, Arango ME. Principios didácticos: de cómo se enseña y aprende en la formación médica. En: Grupo EDUSALUD. Evaluación del currículo del Programa de Medicina de la Universidad de Antioquia. Medellín: Redipe; 2014. p. 198-229.

3. ACNUR [Internet]. Antioquia: ACNUR; 2013 [acceso 2015 Mar 16]. Construyendo soluciones sostenibles. Vereda Granizal, Bello, Antioquia [3 pantallas]. Disponible en: http://www.acnur.org/t3/fileadmin/scripts/doc.php?file=t3/fileadmin/Documentos/ RefugiadosAmericas/Colombia/2013/TSI_Antioquia_VeredaGranizal_septiembre2013

4. Gómez JA, López AA, Rúa H. Estudio de caso: caracterización histórica y sociodemográfica del asentamiento Altos de Oriente, del municipio de Bello. 2010. p. 1-82. Material no publicado. 
5. Agencia Oficiosa en Tutela-Asociaciones de desplazados. Agencia Oficiosa en TutelaCondiciones para que las asociaciones de desplazados interpongan la acción. Sentencia T-025/04. Corte Constitucional de Colombia, Bogotá, D.C., (3 Mar 2004) [acceso 2015 Mar 16]. Disponible en: http://www.corteconstitucional.gov.co/relatoria/2004/t-025-04. $\mathrm{htm}$

6. Galindo LA, Kambourova M, Zuliani L, Villegas ME. Impacto de la visita domiciliaria familiar en el aprendizaje de los estudiantes de medicina en el área de pediatría social. Interface (Botucatu). 2013; 17(46):649-90.

7. Campos FE, Ferreira JR, Fewerwerker L, Sena RR, Campos JJ, Cordeiro H, et. al. Caminos para acercar la formación de profesionales de salud a las necesidades de la atención primaria. Rev Bras Educ Med. 2001; 25(2):1-12.

8. Davini MC. Métodos de enseñanza: didáctica general para maestros y profesores. Buenos Aires: Santillana; 2008.

9. Díaz Barriga F, Hernández Rojas G. Estrategias docentes para un aprendizaje: una interpretación constructivista. México: McGraw- Hill; 1999.

10. Moreno R. Aprendizaje basado en la resolución de problemas. Bol Esc Med. (Pontificia Universidad Católica de Chile). 1996; 25(1):29-32.

11. Marín Londoño B, Tamayo Giraldo G. Currículo integrado: aportes a la comprensión de la formación humana. Pereira: Universidad Católica Popular de Risaralda; 2008.

12. Álvarez de Zayes CM, González Agudelo EM. Lecciones de didáctica general. Bogotá: Cooperativa Editorial Magisterio; 2002.

13. Nussbaum MC. Por qué la democracia necesita de las humanidades. Buenos Aires: Katz Editores; 2010.

14. Ausubel D, Novak J, Hanesian H. Psicología educativa: un punto de vista cognoscitivo. 2a ed. México: Trillas; 1997.

15. Unesco [Internet]. World conference on higher education: higher education in the twenty-first century: vision and action. Paris: Unesco; 1998 [acceso 2015 Mar 16] [33 pantallas]. Disponible en: http://www.unesco.org/education/educprog/wche/declaration spa.htm

16. Martínez EG. El "core currículum": un debate en la educación médica. Educ Educ. (Bogotá). 2006; 9(2):189-96.

17. Restrepo B. Investigación formativa e investigación productiva de conocimiento en la universidad. Nomadas (Bogotá). 2003; (18):195-202.

18. Díaz D. La didáctica universitaria: una alternativa para transformar la enseñanza. Accion Pedagogica (Mérida). 2001; 10(1;2):64-72.

19. Max Neef AM. La transdisciplina: para pasar del saber al comprender. Debates (Medellín). 2003; (36):14-21.

20. Vaideanu G. La interdisciplinariedad en la enseñanza: ensayo de síntesis. Perspectivas [Internet]. 1987 [acceso 2015 Mar 16]; 17(4):531-44. Disponible en: http://unesdoc. unesco.org/images/0007/000782/078248so.pdf

21. Universidad de Antioquia [Internet]. Acuerdo Superior 1 de 1994, 5 de marzo de 1994: por el cual se expide el Estatuto General de la Universidad de Antioquia. Medellín: Universidad de Antioquia; 1994 [acceso 2015 Mar 16]. [44 pantallas]. Disponible en: http://secretariageneral.udea.edu.co/doc/a0001-1994.pdf 
Esta reflexión pedagógica surge de una experiencia académica que se ha desarrollado desde 2004 en la Facultad de Medicina de la Universidad de Antioquia, está enmarcada en el trabajo comunitario con los habitantes de la vereda Granizal (Bello, Antioquia). Se propone avanzar en la estructuración de un currículo integrado teniendo en cuenta las limitaciones del currículo actual. Para lograrlo se plantea la formación profesional de los médicos en los escenarios extramurales diferentes a los tradicionales como el salón de clase y el hospital. Se busca desarrollar en el estudiante habilidades clínicas, reflexivas, pensamiento crítico, actitud investigativa y de comunicación, reforzando la competencia de aprender a aprender y el aprendizaje significativo. Adicionalmente se favorece la interdisciplinariedad, la integración entre básicas-clínicas y teoría-práctica para resolver las necesidades sociales y generar conciencia ético-política y valores de los estudiantes en formación.

Palabras clave: Currículo. Educación. Aprendizaje. Relaciones comunidad-institución.

\section{Necessidades sociais como eixo da formação médica}

A presente reflexão pedagógica começou a partir de uma experiência acadêmica concreta que teve lugar, em 2004, na Faculdade de Medicina da Universidade de Antioquia, e foi desenvolvida com base no trabalho com os moradores da comunidade Granizal, zona rural do município de Bello, Antioquia. Propõe-se mudar o currículo atual (que tem limitações) para um currículo integrado. A principal estratégia para atingir tal mudança é priorizar as comunidades como o foco central da formação, além das aulas e os hospitais. $\mathrm{Na}$ comunidade, os discentes desenvolvem habilidades clínicas de reflexão e investigativas, obtendo a competência de aprender a aprender e a aprendizagem significativa.

Finalmente, na comunidade, o estudante interage com uma equipe interdisciplinar, integra o conhecimento teórico-prático e básico-clínico, além da oportunidade de alcançar consciência ético-política e valores.

Palavras-chave: Currículo. Educação. Aprendizagem. Relações comunidade-instituição.

\section{Medical education based on social needs}

This pedagogical reflection originated from academic experience at the School of Medicine, University of Antioquia, based on community work among the inhabitants of the Granizal community, in the rural zone of the municipality of Bello, Antioquia, which started in 2004. It is proposed to move towards an integrated curriculum, given the limitations of the current one. To achieve this change, the main strategy is to prioritize communities as the central focus of the training, thus going beyond the classroom and the hospital. It is sought to develop reflective clinical skills, critical thinking and investigative and communicative attitudes among the students, thus strengthening their capacity for learning to learn and meaningful learning. Additionally, interdisciplinarity and integration between basic and clinical subjects and between theory and practice are favored, in order to resolve social needs and to create ethical-political awareness and values among the medical students.

Keywords: Curriculum. Education. Learning. Community-institution relations. 
